\title{
Antimycotic Activity of Some Aromatic Plants Essential Oils Against Canine Isolates of Malassezia pachydermatis: An in vitro Assay
}

\author{
Luisa Pistelli ${ }^{1}$, Francesca Mancianti ${ }^{2}$, Alessandra Bertoli ${ }^{1}$, Pier Luigi Cioni ${ }^{1}$, Michele Leonardi ${ }^{1}$ \\ Francesca Pisseri ${ }^{3}$, Linda Mugnaini ${ }^{2}$ and Simona Nardoni ${ }^{*}, 2$ \\ ${ }^{I}$ Dipartimento di Scienze Farmaceutiche, Università di Pisa, Pisa, Italy \\ ${ }^{2}$ Dipartimento di Patologia Animale, Profilassi ed Igiene degli Alimenti, Università di Pisa, Pisa, Italy \\ ${ }^{3}$ Dipartimento di Clinica Veterinaria, Università di Pisa, Italy
}

\begin{abstract}
The antifungal activity and the chemical composition of essential oils (EOs) from some Mediterranean autochthonous plants were investigated against Malassezia pachydermatis, a fastidious opportunistic yeast usually involved in canine external otitis. Minimum inhibitory concentrations (MICs) of Anthemis nobilis, Citrus limon, Citrus paradisi, Illicium verum, Lavandula hybrida, Mentha piperita, Ocimum basilicum, Origanum vulgare, Origanum majorana, Rosmarinus officinalis, Salvia sclarea, Thymus serpillum were assessed by microdilution test; minimum fungicidal activity (MFC) was also determined. O. vulgare, T. serpillum and O. basilicum showed the lowest MIC and MFC values $(0.8 \%)$ followed by $C$. limon and M. piperita (1\%).

EOs from the tested plants showed variable degrees of anti-malassezia activity, putatively related to their chemical composition.

The effectiveness, manageability and pleasant organoleptic properties of $O$. vulgare, T. serpillum, O. basilicum, C. limon and $M$. piperita EOs make them advisable as promising new natural antifungal drugs in the management of $M$. pachydermatis otitis in dog.
\end{abstract}

Keywords: Essential oils activity, malassezia pachydermatis, in vitro sensitivity, microdilution test, gas chromatography-mass spectra, mediterranean plants.

\section{INTRODUCTION}

Essential oils (EOs) are concentrated, hydrophobic substances containing volatile aroma compounds extracted by various processes from different parts of plants. Many common EOs are characterized by healing properties well known in folk medicine since ancient times and still widely used today. The therapeutic value of an EO is held in its composition, which represents a complex make-up of many chemical components with different biological activities. The chemical composition and yield of different EOs show wide variation, depending on the herbal source, chemotype of the plant species, and the analytical methods used [1].

Many studies have demonstrated that extracts from aromatic plants traditionally used in popular medicine, exert antiseptic and inhibitory activities upon filamentous fungi and yeasts [2-4]. The phytotherapeutical use of EOs in veterinary medicine is still poorly substantiated by evidencebased studies, being mainly based on anecdotal experiences of veterinarians, aromatherapists and pet owners.

Malassezia pachydermatis is a normal commensal and occasional pathogen of the skin and mucosae in Carnivora,

*Address correspondence to this author at the Dipartimento di Patologia Animale, Profilassi ed Igiene degli Alimenti, Università di Pisa, Viale delle Piagge, 2, 56100 Pisa; Tel: 00390502216952; Fax: 00390502216941; E-mail: snardoni@unipi.it and represents the most frequently isolated yeast in canine external otitis [5]. Most cases of Malassezia otitis in the dog are associated with concurrent skin disorders or systemic diseases. Treatment is generally based on topical administration of azoles or nystatin, combined with antibiotics and glucocorticoids [6]. Many dogs affected by Malassezia require regular maintenance therapy to prevent relapse, which is common when predisposing factors are not identified or corrected.

The factors involved in the transition from commensalism to parasitism by $M$. pachydermatis in dogs are not fully understood even if it is well stated that the interface between commensalism and pathogenicity in Malassezia infections is a fine balance, in which both the regulation of host immune response and the activity of the fungus are strictly involved [7].

Among the multitude of EOs, tea tree oil (Melaleuca alternifolia) is the most extensively tested against several microbial agents. Some studies deal with the effectiveness versus Malassezia yeasts responsible for both canine [8] and human infections $[9,10]$. In veterinary medicine attention should be paid to otologic use of tea tree oil due to its toxicity after instillation into the ear [11], consequently the search for alternative products is recommended.

In recent years, the interest in selecting products from landscape plants that are sustainable has increased and some data are available for malassezia species of human interest 
indicating extract of Citrus aurantifolia as the most active against Malassezia furfur [12].

Aim of the present study was to evaluate the in vitro antiM. pachydermatis activity of EOs from Anthemis nobilis, Citrus limon, Citrus paradisi, Illicium verum, Lavandula hybrida, Mentha piperita, Ocimum basilicum, Origanum vulgare, Origanum majorana, Rosmarinus officinalis, Salvia sclarea, Thymus serpillum using a microdilution test. All the EOs were supplied by Flora srl (Lorenzana, Pisa, Italy).

Volatile constituents of each EO were identified by GCMS analysis, in order to investigate the relation between chemical composition and biological activity as antifungal agents. GC-MS analysis was accomplished with a HP-5890 Series II instrument equipped with a HP-Wax and HP-5 capillary columns (both $30 \mathrm{~m} \mathrm{X} 0.25 \mathrm{~mm}, 0.25 \mu \mathrm{m}$ film thickness), working with the following temperature program: $60{ }^{\circ} \mathrm{C}$ for $10 \mathrm{~min}$, rising at $5{ }^{\circ} \mathrm{C} / \mathrm{min}$ to $220^{\circ} \mathrm{C}$. The injector and detector temperatures were maintained at $250{ }^{\circ} \mathrm{C}$; carrier gas nitrogen $(2 \mathrm{~mL} / \mathrm{min})$; detector dual, FID; split ratio 1:30. The volume injected was $0.5 \mu \mathrm{L}$.

GC-MS analysis was performed with a Varian CP-3800 gas-chromatography equipped with HP-5 capillary column (30 m X $0.25 \mathrm{~mm}$; film thickness $0.25 \mu \mathrm{m}$ ). Analytical conditions: injector and transfer line temperature $220^{\circ} \mathrm{C}$ and $240{ }^{\circ} \mathrm{C}$, respectively; oven temperature programmed $220{ }^{\circ} \mathrm{C}$ and $240{ }^{\circ} \mathrm{C}$ from $60{ }^{\circ} \mathrm{C}-240{ }^{\circ} \mathrm{C}$ at $3{ }^{\circ} \mathrm{C} / \mathrm{min}$.; carrier gas helium at a flow rate of $1 \mathrm{~mL} / \mathrm{min}$.; split ratio 1:30. Mass spectra were recorded at $70 \mathrm{eV}$. The acquisition mass range was $\mathrm{m} / \mathrm{z} 30-300$ at a scan rate of $1 \mathrm{scan} / \mathrm{sec}$.

Identification of the constituents was based on comparison of the retention time with those of authentic samples, comparing their linear indices relative to a series of $n$ alkanes (C8-C23). Further identifications were also made possible by the use of a homemade library of mass spectra built up from pure substances and components of known oils, and MS literature data (NIST 2000, ADAMS).

EOs antifungal activity was tested against five clinical isolates of $M$. pachydermatis, obtained from recurrent cases of canine external otitis. M. pachydermatis reference strain CBS 1879 was used as reference strain. Diagnosis of Malassezia otitis was based on history, microscopic and otoscopic examination. The ceruminous discharge was collected with a sterile-tipped applicator from the junction of the vertical and horizontal ear canal for fungal culture and the samples were promptly seeded onto Sabouraud Dextrose Agar added with $0.5 \%$ of chloramphenicol and cycloheximide (DID, Milano, Italy) and mDixon agar (3.6\% malt extract, $0.6 \%$ peptone, $2 \%$ desiccated ox-bile, $1 \%$ Tween 40 , $0.2 \%$ glycerol, $0.2 \%$ oleic acid, $1.2 \%$ agar). The plates were incubated at $30^{\circ} \mathrm{C}$ for 7 days and processed as previously reported [13]. M. pachydermatis strains apparently lipiddependent were identified by serial transfers on a lipid-free culture medium. The Tween assimilation test as described by Guého et al., (1996) [14] and catalase activity were performed as additional tests both to confirm the identification, and to exclude the presence of other Malassezia species. Subcultures were inoculated on mDixon agar plates, to obtain pure isolates.

For all in vitro studies, $A$. nobilis s. $71565, C$. limon s. 90660, C. paradisi s. 60221, I. verum s. 70487, L. hybrida s. 80241, M. piperita s. 80059, O. basilicum s. 70486, O. vulgare s. $60326, O$. majorana s. $80717, R$. officinalis s.60734, S. sclarea s.80193, T. serpillum s. 90017 and sweet almond oil s.70312 of EOs were assayed.

Stock solutions of EOs were prepared in sweet almond oil (Prunus dulcis Mill. Flora Srl., Lorenzana, Pisa, Italy) and diluted to obtain concentrations ranging from $0,6 \%$ to $10 \%$. The dilutions were chosen considering that EOs in vivo maximum dilution for dermatologic administration is $20 \%$. Considered both the lack of related reference data and the peculiar morpho-physiological features of external auditory canal, $10 \%$ was selected as starting dilution for the assay to avoid the risk of ear swelling and inflammation.

The effectiveness of EOs was assessed by means of a microdilution test carried out as described by Hammer et al., (1997) [9], modified using a semisolid mDixon agar with $0.6 \%$ agar instead of solid mDixon. This medium was adopted to ensure the optimal growth of Malassezia strains, while the use of a semisolid medium was more suitable to the lipophylic nature of EOs tested, to ensure a better mutual contact between yeast cells and the different EOs during the assays, as previously described [15]. Control cultures were achieved with medium alone, and with medium supplemen-

Table 1. Chemical Composition of the Tested Essential Oils

\begin{tabular}{|c|c|c|c|c|c|c|c|c|c|c|c|}
\hline \multirow{2}{*}{ Chemical Class } & \multicolumn{11}{|c|}{ Relative Percentage Area (\%) } \\
\hline & o.v. & o.b. & T.s. & C.I. & M.p. & R.o. & O.m. & I.v. & C.p. & L.h. & S.s \\
\hline Oxygenated monoterpenes & 58,1 & 68,8 & 80,3 & 4,9 & 86,5 & 46,1 & 63,0 & 95,1 & 0,9 & 73,2 & 91,2 \\
\hline Total monoterpenes & 92,7 & 71,1 & 96,5 & 97,4 & 93,3 & 96,6 & 88,4 & 99,9 & 98,0 & 84,7 & 93,9 \\
\hline Oxygenated sesquiterpenes & 0,2 & 7,9 & 0,4 & & 0,4 & 0,2 & 0,9 & & 0,1 & 1,5 & 1,2 \\
\hline Total sesquiterpenes & 6,0 & 27,6 & 2,6 & 2,6 & 5,6 & 2,5 & 5,8 & & 1,2 & 11,7 & 3,2 \\
\hline Other & 1,0 & & & & 0,3 & & 0,2 & & 0,9 & 1,8 & 0,5 \\
\hline
\end{tabular}

O.v: Origanum vulgare; O.b.: Ocimum basilicum; T.s.: Thymus serpillum; C.I.:Citrus limon; M.p.:Mentha piperita; R.o.: Rosmarinum officinalis; O.m.: Origanum majorana; I.v.:Illicium verum; C.p.: Citrus paradisi; L.h.: Lavandula hybrida; S.s.: Salvia sclarea. 
Table 2. Main Constituents of the Tested Essential Oils

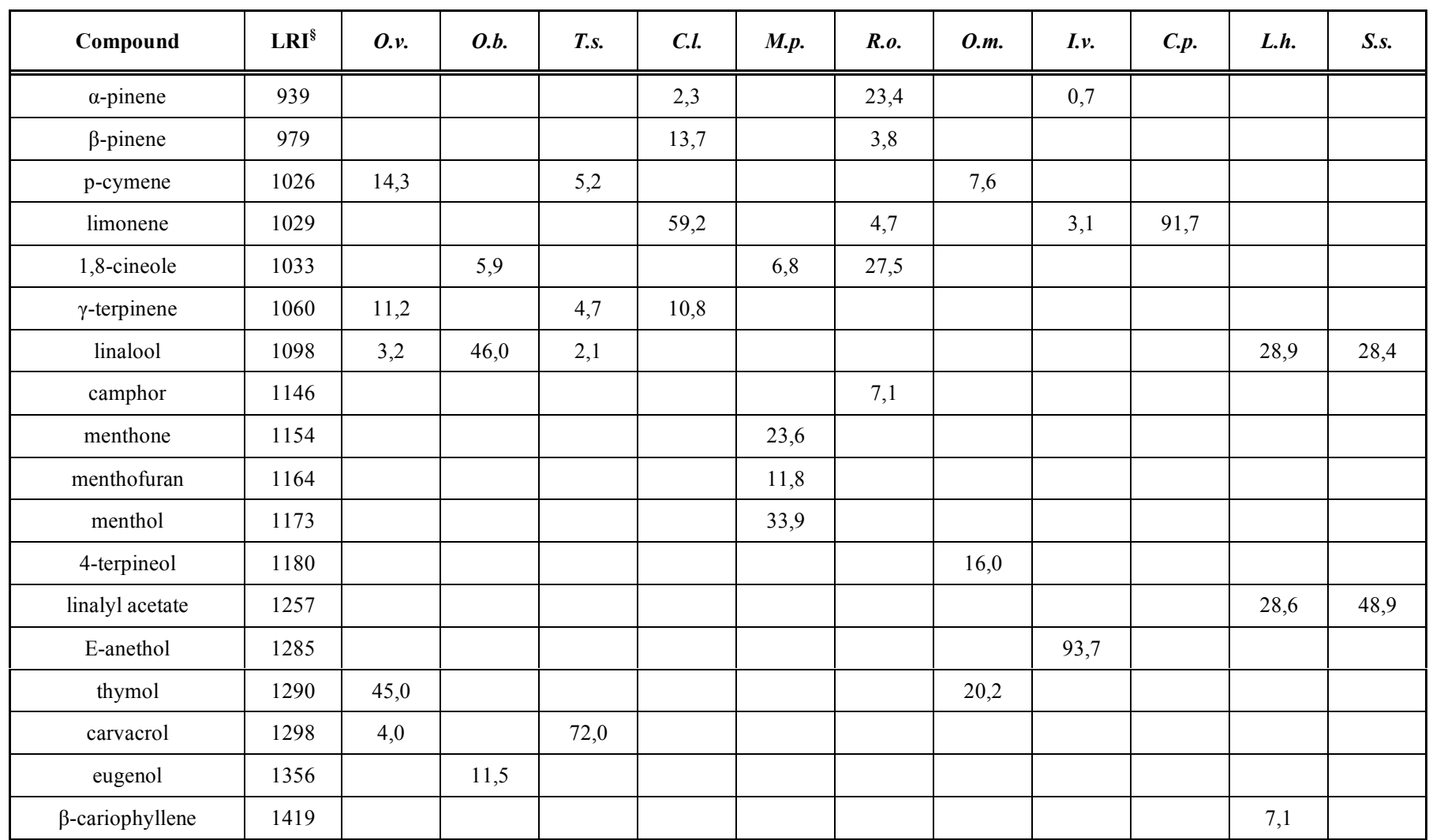

${ }^{\S}$ Linear Retention Index calculated on the basis of retention time of a mixture of $n$-alkanes $\left(\mathrm{C}_{8}-\mathrm{C}_{30}\right)$

O.v: Origanum vulgare; O.b.: Ocimum basilicum; T.S.: Thymus serpillum; C.I.: Citrus limon; M.p.: Mentha piperita; R.o.: Rosmarinum officinalis; O.m.: Origanum majorana; I.v.: Illicium verum; C.p.: Citrus paradisi; L.h.: Lavandula hybrida; S.s.: Salvia sclarea

ted with $50 \%$ of sweet almond oil, respectively. Furthermore, the yeasts were tested against ketoconazole by E-test (AB Biodisk, Solna, Sweden), as described by Nijima et al., (2010) [16]. Cultures were incubated at $30^{\circ} \mathrm{C}$ for $24-72 \mathrm{~h}$, until a full development of the yeasts in control wells was noticeable. After incubation, yeasts inocula were harvested by centrifugation, gently washed in fresh mDixon broth, then inoculated on mDixon agar plates to monitor killing of the yeasts. All tests were performed in quadruplicate.

All analyzed EOs showed a high percentage of total monoterpene derivatives $(>85 \%$, with the highest value in $I$. verum), while only the EO obtained from $O$. basilicum was characterized by a lower percentage of these compounds $(71,1 \%)$. In detail, the chemical composition of the tested EOs was characterized by a high amount of oxygenated monoterpenes. The two Citrus species analyzed had an opposite profile with a predominance of hydrocarbon monoterpenes (97,1\% in C. paradisi and $92,5 \%$ in C. limon). The ratio between the oxygenated and non-oxygenated monoterpenes for the $R$. officinalis essential oil was near to 1. The presence of sesquiterpene derivatives ranged from $0,0 \%$ (I. verum) to $27,6 \%$ (O. basilicum). The chemical composition of tested EOs and main constituents are reported in Tables $\mathbf{1}$ and $\mathbf{2}$, respectively.

The plates were daily observed and effects were evaluated at $72 \mathrm{~h}$ p.i., when control wells showed a good fungal development. All EOs except $S$. sclarea inhibited the growth of M. pachydermatis tested strains at different concentrations (Table 3).

Table 3. Minimum Inhibition Concentration (MIC) and Minimum Fungicidal Concentration (MFC) of the Tested Essential Oils

\begin{tabular}{|c|c|}
\hline Essential Oil & MIC and $-\mathbf{M F C}(\mathbf{\%} \mathbf{v} / \mathbf{v})$ \\
\hline \hline Citrus limon & 1 \\
\hline Citrus paradisi & 1,3 \\
\hline Illicium verum & 1,3 \\
\hline Lavandula hybrida & 4 \\
\hline Mentha piperita & 1 \\
\hline Ocimum basilicum & 0,8 \\
\hline Origanum vulgare & 0,8 \\
\hline Origanum majorana & 1,3 \\
\hline Rosmarinus officinalis & 1,3 \\
\hline Salvia sclarea & $>10$ \\
\hline Thymus serpillum & 0,8 \\
\hline
\end{tabular}

T. serpillum, $O$. vulgare and $O$. basilicum had the highest MICs $(0.8 \%)$, while $4 \%$ L. hybrida inhibited yeasts' growth. Washed and re-seeded inocula failed to yield a 
growth, showing same values for MIC and MFC for all the strains and for all the oils tested. The MIC for ketoconazole was $<0,03 \mu \mathrm{g} / \mathrm{ml}^{-1}$ for all yeasts isolates.

Several publications on antifungal activity of EOs are reported in literature. In the present paper EOs obtained from $T$. serpillum, $O$. vulgare, $O$. basilicum, $C$. limon and $M$. piperita showed a good antifungal action against $M$. pachydermatis, inhibiting mycotic development at high dilution. Their good biological activity may be related to the chemical composition. T. serpillum was characterized by an high percentage of oxygenated monoterpene derivatives $(80,3 \%)$, in particular carvacrol was the more represented compound of this EO $(72 \%)$, while $\gamma$-terpinene and $\mathrm{p}$ cymene were present in minor percentages $(4,7 \%$ and $5,2 \%$, respectively). The high biological activity observed in $T$. serpillum EO is in agreement with the literature, even if the chemical composition showed some differences in the amount of thymol. At this regard, Soković et al., (2009) [2] reported a very strong antifungal activity for this plant due to the presence of both thymol and carvacrol. Thymol was the main constituent of $O$. vulgare EO (45\%), with lower amounts of $\mathrm{p}$-cimene $(14,3 \%)$ and $\gamma$-terpinene $(11,2 \%)$. Antimalassezia activity of oregano was assessed by Lee and Lee (2010) [12] also, confirming our results. The EO profile of $O$. basilicum was characterized by an high percentage of linalool (46\%) and eugenol (11,5\%) while 1,8-cineole and $\beta$ pinene were less represented. The in vitro activity of $O$. basilicum was assayed by Saggiorato et al., (2009) [17], who reported the efficacy of this EO as a natural fungicide. $C$. limon EO contained an high percentage of the hydrocarbon monoterpene derivative limonene (59,2\%). Several Authors have attributed the antifungal activity of $C$. limon to the presence of volatile compounds such as limonene and linalool. This activity may be determined by single major compounds or by synergistic or antagonistic effect of various components [18]. C. limon EO therefore contains $\gamma$-terpinene and $\beta$-pinene, also. Finally the EO obtained from $M$. piperita was characterized by a high percentage of menthol $(33,9 \%)$ and menthone (23,6\%). Soković et al., (2009) [2] reported for these two derivatives a very strong antifungal activity. Even if potential antifungal effects of some compounds from plants have recently attracted attention, there is scanty scientific information about anti-malassezia properties of herbal remedia. To the best of our knowledge, in vitro sensitivity of $M$. pachydermatis has been scarcely investigated in veterinary medicine $[4,19]$. It is well stated that malassezia acts as an opportunist agent, so identification and correction of predisposing diseases is often essential for successful management, although many dogs with Malassezia otitis require regular maintenance therapy. Predisposing factors such as all dermatologic disorders resulting in alteration of skin surface environment (seborrhea, otitis) are responsible for relapsing infection and repeated administration of conventional antifungal drugs may cause side effects. EOs show eudermic, lenitive and anti-inflammatory activities and may contribute to skin healing. Moreover the use of compounds with a pleasant smell is appreciated by the owners, fighting the strong odour of rancid fat, characteristic of malassezia skin disorders. For these reasons the choice of EOs could be an intriguing alternative.
There are few experienced herbal vets, so safe and effective coordination in a holistic manner is not common. Off-the-shelf herbal medicines can therefore be dangerous if there is no skilled professional herbal vet overseeing the entire input given to the patient. Furthermore attention should be paid in EOs' selection in the treatment of external and middle ear infections. It is important to select new compounds with neither residual nor toxic properties and to remember that herbs and conventional medicines can clash dangerously or can summate with a risk of serious overdose.

\section{CONFLICT OF INTEREST}

None declared.

\section{ACKNOWLEDGEMENT}

None declared.

\section{REFERENCES}

[1] Jardim CM, Jham GN, Dhingra OD, Freire MM. Composition and antifungal activity of the essential oil of the Brazilian Chenopodium ambrosioides L. J Chem Ecol 2008; 34: 1213-8.

[2] Soković MD, Vukojević J, Marin PD, Brkić DD, Vajs V, van Griensven LJLD. Chemical composition of essential oils of Thymus and Mentha species and their antifungal activities. Molecules 2009; 14: 238-49.

[3] Palmeira de Oliveira A, Salgueiro L, Palmeira de Oliveira R, et al. Anti-Candida activity of essential oils. Mini Rev Medic Chem 2009; 14: 1292-305.

[4] Rukayadi Y, Hwang JK. In vitro antimycotic activity of xanthorrhizol isolated from Curcuma xanthorrhiza Roxb. against opportunistic filamentous fungi. Phytother Res 2007; 21: 434-8.

[5] Campbell JJ, Coyner KS, Rankin SC, Lewis TP, Schick AE, Shumaker AK. Evaluation of fungal flora in normal and diseased canine ears. Vet Dermatol 2010; 21: 619-25.

[6] Bond R. Superficial veterinary mycoses. Clin Dermatol 2010; 28, 226-36.

[7] Chen TA, Hill PB. The biology of Malassezia organisms and their ability to induce immune responses and skin disease. Vet Dermatol 2005; 16: 4-26.

[8] Weseler A, Geiss HK, Saller R, Reichling J. Antifungal effect of Australian tea tree oil on Malassezia pachydermatis isolated from canines suffering from cutaneous skin disease. Schweiz Arch Tierheilkd 2002; 144: 215-21.

[9] Hammer KA, Carson CF, Riley TV. In vitro susceptibility of Malassezia furfur to the essential oil of Melaleuca alternifolia. J Med Vet Mycol 1997; 35: 375-7.

[10] Hammer KA, Carson CF, Riley TV. In vitro activities of econazole, miconazole, and Melaleuca alternifolia (tea tree) oil against Malassezia species. Antimicrob Agents Chemother 2000; 44, 4679.

[11] Zhang SJ, Robertson D. A study of tea tree oil ototoxicity. Audiol Neurootol 2000; 5: 64-8.

[12] Lee JH, Lee JS. Chemical composition and antifungal activity of plant essential oils against Malassezia furfur. Kor J Microbiol Biotechnol 2010; 38: 315-21.

[13] Nardoni S, Mancianti F, Corazza M, Rum A. Occurrence of Malassezia species in healthy and dermatologically diseased dogs. Mycopathologia 2004; 157: 383-8.

[14] Guého E, Midgley G, Guillot J. The genus Malassezia with description of four new species. Antonie Van Leeuwenhoek 1996; 69: $337-55$.

[15] Nardoni S, Bertoli A, Pinto L, Mancianti F, Pisseri F, Pistelli L. In vitro effectiveness of tea tree oil against Trichophyton equinum. J Mycol Méd 2010; 20: 75-9.

[16] Nijima M, Kano R, Nagata M, Hasegawa A, Kamata H. An azoleresistant isolate of Malassezia pachydermatis. Vet Microbiol 2011; 149: 288-90. 
[17] Saggiorato AG, Gaio I, Treichel H, de Oliveira D, Cichoski AJ, Cansian RL. Antifungal activity of basil essential oil (Ocimum basilicum L.): evaluation in vitro and on an Italian-type sausage surface. Food Bioprocess Technol 2009; 5(1): 378-84. DOI: 10.1007/s11947-009-0310-z.

[18] Viuda-Martos M, Ruiz-Navajas Y, Fernandez-Lopez J, PerezAlvarez J. Antifungal activity of lemon (Citrus lemon L.), mandarin (Citrus reticulata L.), grapefruit (Citrus paradisi L.) and orange (Citrus sinensis L.) essential oils. Food Control 2008; 19: 1130-8.

[19] Brodin K, Alahyar H, Hedner T, Sterner O, Faergemann J. In vitro activity of Artemisia abrotanum extracts against Malassezia spp. Candida albicans and Staphylococcus aureus. Acta Derm Venereol 2007; 87: 540-2.

(C) Pistelli et al.; Licensee Bentham Open.

This is an open access article licensed under the terms of the Creative Commons Attribution Non-Commercial License (http://creativecommons.org/licenses/by$\mathrm{nc} / 3.0 /$ ), which permits unrestricted, non-commercial use, distribution and reproduction in any medium, provided the work is properly cited. 